\title{
Modeling and Experimental Analysis of an In-body Area Nanonetwork
}

\author{
Valeria Loscrí \\ FUN Team \\ INRIA Lille-Nord Europe \\ Lille, France
}

\author{
Ladislau Matekovits ${ }^{\dagger}$ \\ Department of Electronics and \\ Telecommunications \\ Politecnico di Torino \\ Torino, Italy
Anna Maria Vegni ${ }^{\S}$
Roma TRE University \\ Department of Engineering \\ Rome, Italy
}

\author{
Ildiko Peter $\ddagger$ \\ Department of Applied \\ Science and Technology \\ Politecnico di Torino \\ Torino, Italy
}

\begin{abstract}
Nanotechnology is gaining more and more consensus in several application fields, comprised in-body applications. Innovative therapies and diagnostic approaches are based on the use of injections or oral delivery of nanoparticles. The acceleration of this type of development makes very urgent a deeply and accurate analysis of the interaction between the nanoparticles and the biological fluid where the nanoparticles are immersed.

In this paper, we focus on Titanium ( $\mathrm{T} i$ and its alloy (e.g., $\mathrm{TiO}_{2}$ ) systems, given their specific features in terms of density, lack of cytotoxic effects, etc.. Specifically, we design and study an in-body system by characterizing the emission and diffusion processes. Further, we propose an experimental study of the morphology and other important characteristics (e.g. the $\mathrm{pH}$ of the particles, and the dielectric constant of the solution) of nano- $\mathrm{TiO}_{2}$ systems when immersed in a Ringer
\end{abstract}

\footnotetext{
*Valeria Loscrí is with INRIA Lille-Nord Europe, France. Email: valeria.loscri@inria.fr

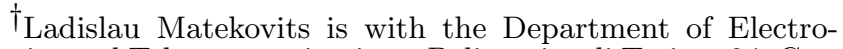
nics and Telecommunications, Politecnico di Torino, 24, C.so Duca degli Abruzzi, 10129 Torino, Italy.

Email: ladislau.matekovits@polito.it

$\ddagger$ Ildiko Peter is with the Department of Applied Science and Technology, Politecnico di Torino, 24, C.so Duca degli Abruzzi, 10129 Torino, Italy. Email: ildiko.peter@polito.it

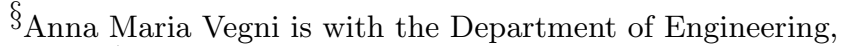
COMLAB Telecommunication Lab, Roma TRE University, Via Vito Volterra, 62, 00146 Rome, Italy.

Email: annamaria.vegni@uniroma3.it
}

solution, in order to derive important information related to their potential toxicity inside the human body.

\section{Categories and Subject Descriptors}

I.6 [Simulation and Modeling]: Miscellaneous; I.6.5 [Model Development]: Modeling methodologies-complexity measures, performance measures

\section{General Terms}

Theory, Experimentation

\section{Keywords}

In-body area nanonetwork, titanium nanoparticles, Ringer solution, nanoparticle emission and diffusion

\section{INTRODUCTION}

Nanotechnology is a new research field that finds applications in several areas. In nanomedicine, the use of nanodevices (i.e. nanorobots and nanosensors, opportunely functionalized or inner drugged with medicament) may not only improve the therapeutic index of drugs by improving their effectiveness, and increasing their tolerability in the body by interfering at a minimum level with the immune system [1], but also for diagnostic purposes $[2,3,4]$ even if the related security and privacy issues present very important challenges [5].

Different types of nanoparticles can be exploited for nanomedicine applications, and are constructed from a huge range of organic and inorganic materials, such as emulsions, Solid Lipid Nanoparticles (SLNs), gold nanoparticles, silver nanoparticles, titanium dioxide $\mathrm{Ti}_{2}$ nanoparticles, dendrimers, Carbon Nano-Tubes (CNTs), etc. just to mention the main types [6]. All these materials can be used as carriers for localized Drug Delivery Systems (DDSs) and for diagnostic scopes when opportunistically functionalized. In the first case, the aim is to provide a locally controlled drug concentration, where the medication is needed [2]. The second case is to provide information about in-body conditions, 
by opportunistically measuring specific parameters and/or chemical concentrations, etc. [3].

Noble metals, like silver and gold, are largely used for the design of nanoparticles in the field of sensing applications, as well as acting as carriers of medicament molecules. Particularly, colloidal silver has been used as an antibacterial agent by weakening DNA replication and inactivating proteins, while gold has low toxicity to biological systems, and so results inefficient for antibiotic therapy [7].

In addition, nanoparticles are also engineered to provide sustained drug release [8, 9], especially beneficial for chronic therapies. In [10], the authors use gold nanoparticles for diagnostic and drug delivery applications by exploiting chitosan, with the dual purpose of $(i)$ acting as a reducing agent in the synthesis of gold nanoparticles, and also (ii) promoting the penetration and uptaking the peptide hormone insulin across the mucosa.

For both DDS and diagnostic applications, common methods adopted include $(a)$ inhalation, $(b)$ intravenous and intramuscular injection and $(c)$ ingestion/oral [11]. In all these methods, the most important thing is to characterize the interaction between the in-body solution and the specific nanoparticles.

The contribution of this paper is twofold: $(i)$ the characterization of the $\mathrm{TiO}_{2}$ nanoparticles emission, and diffusion processes, and $(i i)$ an experimental analysis of the $\mathrm{TiO}_{2}$ nanoparticles in a biologic-like fluidic medium, in order to infer main features as $\mathrm{pH}$, diffraction patterns and dielectric constant.

Notice that the choice of $\mathrm{TiO}_{2}$ nanoparticles is due to their benefits for biomedical applications. Indeed, among other existing systems, because of their low density, excellent corrosion resistance, lack of cytotoxic effects, good mechanical properties, $T i$ and its alloys, $\mathrm{TiO}_{2}$ are well established materials for biomedical application as orthopedic and dental implants, cardiovascular stents, joint replacement [12], [13, $14,15,16]$. Addition of a controlled drug delivery characteristic to $\mathrm{TiO}_{2}$ nanoparticles could enlarge its potential use in biomedical fields.

This paper is organized as follows. In Section 2 we present our general (and not specific to materials discussed later in the paper) nanoparticulate model suitable both for injection of tablets and liquids containing nanoparticle concentration. We highlight the main processes that occur when a concentration of nanoparticles (i.e. $\mathrm{Ti}_{2}$ nanoparticles in our case) is introduced in what we name here "compartment" to indicate a closed volume containing a biological fluid. Specifically, we detail the laws characterizing the emission/ transmission ${ }^{1}$ and diffusion processes. In Section 3 we describe the details of the experiment we dealt in order to infer the main characteristics of the interactions between the nanoparticles and the biological fluid. Finally, conclusions are drawn at the end of the paper.

\footnotetext{
${ }^{1}$ In this paper, the terms emission and transmission are interchanged.
}

\section{CHARACTERIZATION OF THE IN-BODY PHASES}

In this section, we describe the two main processes that characterize the pharmacokinetics model we considered in this work, namely the emission and the diffusion.

\subsection{Nanoparticle emission process}

In our model, we rely on the pharmacokinetics laws, which provide a mathematical basis for analyzing how the human body reacts to the administration of "external substances" (i.e. a fluid with a given nanoparticle concentration). Pharmacokinetics considers the rate at which drug concentrations change in the body, and includes the kinetics of absorption, distribution, metabolism and excretion of a drug.

There exist three approaches of pharmacokinetics modeling i.e., (i) compartmental, (ii) physiological, and (iii) modelindependent. The first is an empirical approach, where the compartment can represent a body/organ volume. Usually one or two compartments are used. The physiological model identifies the compartments with actual body spaces. The model is more complex than the compartmental models; it is largely used for predictions, and is more suitable for clinical therapy, and when changing situations like alterations of flow rates due to conditions such as swelling, or fever occur. Finally, the model-independent approach is the most recent, but does not provide any specific information about how the drug is distributed.

In this paper, we rely on one compartment model for oral drug administration, so that we can model a compartment with a biological fluid (e.g. the Ringer solution as described in Section 3) into which a flow of nanoparticles is added by a rapid single dose. Notice that the multi-particulate DDSs show reproducible pharmacokinetics behavior than conventional (monolithic) formulations; this means that after disintegration, which occurs within a few minutes (often even within seconds), the individual nanoparticles are released within the "compartment".

After such a period (i.e., $t_{1}[\mathrm{~s}]$ ), we assume that the nanoparticle concentration i.e., $C_{N P}\left(t_{1}\right)$, is uniform in the "compartment", and has reached a maximum value. The analysis of this model relies on a simple mass balance compliant with the dissolution kinetics of $\mathrm{Ti}_{2}$ nanoparticles [17] that have been used in the experiments described in Section 3.

The mass balance model that provides the nanoparticle transmission rate in the compartment model is expressed as

$$
r_{T x}(t)=\frac{d C_{N P}(t)}{d t}=k_{d} c_{i n}-k_{e} C_{N P}(t),
$$

where $C_{N P}(t)$ is the nanoparticle concentration behavior along time, $c_{i n}$ is the initial nanoparticle concentration, $k_{d}$ $\left[\min ^{-1}\right]$ and $k_{e}\left[\mathrm{~h}^{-1}\right]$ are the first-order dissolution and elimination rate constants, respectively. The solution of (1) is obtained by integration:

$$
C_{N P}(t)=\frac{k_{d}}{k_{d}-k_{e}} \cdot C_{0}\left[\exp \left(-k_{e} t\right)-\exp \left(-k_{d} t\right)\right]
$$

where $C_{0}\left[\mathrm{~L}^{-1}\right]$ represents the amount of nanoparticles (dose) at zero concentration. The peak of nanoparticle concentra- 
tion in the compartment corresponds to $C_{N P}\left(t=t_{1}\right)$, i.e.,

$$
C_{N P}\left(t_{1}\right)=\frac{N N P}{V},
$$

with $N N P$ as the quantity of nanoparticles in the volume $V$. Notice that $V$ has no direct physiological meaning, but corresponds to the apparent volume of distribution, defined as that volume of plasma in which the total amount of nanoparticles in the compartment would be required to be dissolved in order to reflect the nanoparticles concentration attained in plasma.

Fig. 1 depicts the behavior of nanoparticles in the compartment i.e., the nanoparticle concentration versus time. We assumed $C_{0}=100\left[\mathrm{~L}^{-1}\right], k_{e}=0.0578\left[\mathrm{~h}^{-1}\right]$, and different values of dissolution constant i.e., $k_{d}=(0.03,0.05,0.07)$ $\left[\mathrm{min}^{-1}\right]$. The value of $k_{d}=0.03$ corresponds to the dissolution kinetics constant of the $\mathrm{TiO}_{2}$ nanoparticles [18]. We notice that the concentration increases faster for higher values of dissolution constant, while it decreases very slowly due to typical value of elimination constant, that is

$$
k_{e}=\frac{\ln (2)}{t_{1 / 2}},
$$

where $t_{1 / 2}[\mathrm{~h}]$ is the elimination half-life, that is the time required for the concentration of the nanoparticles to reach half of its original value (i.e., $t_{1 / 2}=12 \mathrm{~h}$ ).

Notice that the behavior of nanoparticle concentration in the compartment reflects that of a pulse-based modulation scheme [19], where a transmitter nanomachine instantaneously releases a pulse of molecules. Indeed, due to the nano-scale size of nanoparticles approximable to that of molecules, we can consider the nanoparticle concentration behavior as a spike, followed by a slow decrease of concentration, as depicted in Fig. 1.

The time instant when the nanoparticle concentration reaches the peak is $t_{1}[\mathrm{~min}]$, and the analytical expression can be obtained by derivation of (2), posed equal to zero, such as

$$
\frac{d C_{N P}(t)}{d t}=\frac{k_{d} C_{0}}{k_{d}-k_{e}} \cdot \frac{d\left[\exp \left(-k_{e} t\right)-\exp \left(-k_{d} t\right)\right]}{d t}=0,
$$

from which follows that

$$
t_{1}=\frac{1}{\left(k_{d}-k_{e}\right)} \cdot \ln \left(\frac{k_{d}}{k_{e}}\right),
$$

where $k_{d} \neq k_{e}$. This expression represents the time instant for which the concentration of emitted nanoparticles reaches its global maximum, and can be interpreted as the nanoparticle concentration delay [min], depending on the values of dissolution and elimination constants. Notice that this delay represents the time for the whole dose of nanoparticles to be emitted outside the tablet; for higher values of the dissolution constant $k_{d}$, the delay decreases, meaning that the nanoparticle concentration reaches the peak faster.

Fig. 2 depicts the nanoparticle transmission delay, versus the dissolution constant. It is intuitive that higher the dissolution rate (i.e., $k_{d}$ ), lower will be the transmission delay occurring from the emission process at the transmitter (i.e., the tablet) up to the diffusion process, where the maximum nanoparticle concentration has been delivered.

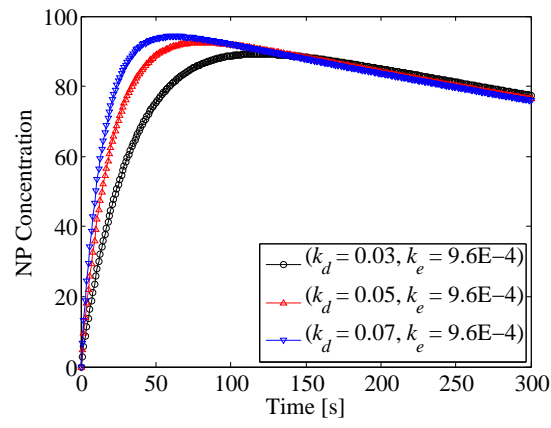

Figure 1: Nanoparticle concentration within the compartment model, for different values of the dissolution constant. Higher the dissolution constant, faster the increase of concentration.

Another feature is the nanoparticle concentration width $t_{w}$ [min] that represents the time interval from $t_{1}$ up to $t_{2}$, where $t_{2}$ is that value corresponding to an amplitude of NP concentration greater than half of its maximum value, that is:

$$
C_{N P}\left(t_{2}\right)=\frac{C_{N P}\left(t_{1}\right)}{2},
$$

which becomes

$$
\frac{N N P}{2 V}=\frac{k_{d}}{k_{d}-k_{e}} C_{0}\left[\exp \left(-k_{e} t_{2}\right)-\exp \left(-k_{d} t_{2}\right)\right],
$$

and simplified provides the value of $t_{2}$ [min], that is

$$
t_{2}=-\frac{1}{k_{e}-k_{d}} \ln \left(\frac{N N P}{2 V} \cdot \frac{k_{d}-k_{e}}{k_{d} C_{0}}\right) .
$$

From (9), we can obtain the expression of the nanoparticle concentration width $t_{w}[\mathrm{~min}]$ as

$$
t_{w}=t_{2}-t_{1},
$$

where by substituting (6) and (9), we obtain:

$$
t_{w}=-\frac{1}{k_{e}-k_{d}} \ln \left(\frac{N N P}{2 V} \cdot \frac{k_{d}-k_{e}}{k_{d} C_{0}}\right)-\frac{1}{k_{d}-k_{e}} \ln \left(\frac{k_{d}}{k_{e}}\right),
$$

which becomes

$$
t_{w}=\frac{1}{\left(k_{d}-k_{e}\right)} \cdot \ln \frac{N N P}{C_{0} V} .
$$

\subsection{Nanoparticle diffusion process}

The diffusion process can be studied by distinguishing two cases i.e., ( $i)$ the ideal, and ( $i i)$ non-ideal case. In the first scenario, the nanoparticle concentration is assumed as homogeneous and no nanoparticle is lost during the diffusion. On the other hand, in the non-ideal case a portion of nanoparticles is lost and cannot reach a destination point.

In an ideal case, the concentration is assumed homogeneous and the residence time attains a mean reference value. Unfortunately, in a real reactor the nanoparticle fluid spends a different time inside the reactor, depending on specific characteristics of the compartment. The ideal case that models the compartment can be represented through a bioreactor. A bioreactor is a reactor that utilizes either a living organism 


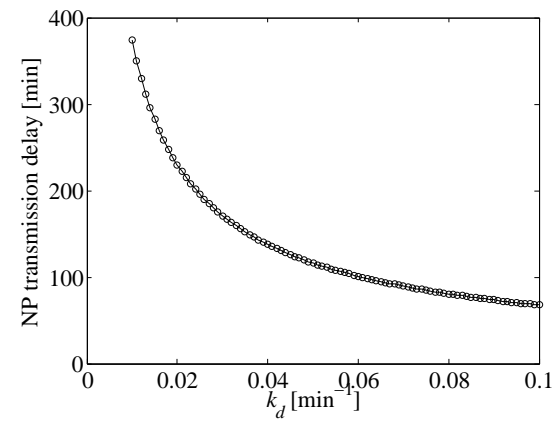

Figure 2: Transmission delay of nanoparticle concentration within the compartment model, versus different values of the dissolution constant. Higher the dissolution constant, lower the nanoparticle transmission delay.

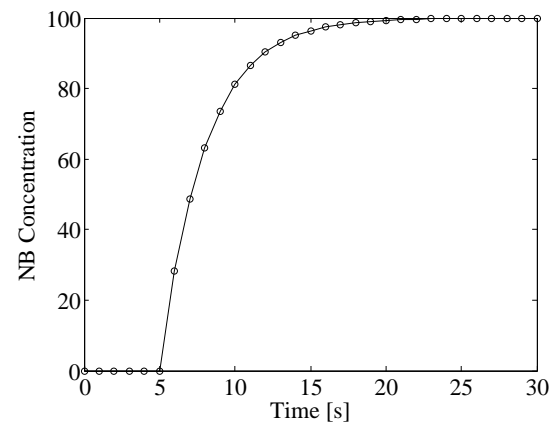

Figure 3: Binding concentration within the compartment model, which occurs for $t>5 \mathrm{~s}$, and increases until reaching the maximum value $N B$.

or one or more enzymes from a living organism to accomplish a certain chemical transformation.

For the non-ideal case, from (1), the nanoparticle transmission rate $r_{T}(t)$ can be identified with the nanoparticle concentration flux $J_{T}(x, t)$ at the output of the tablet i.e., for $x=x_{T}$. The particle concentration flux $J_{T}(x, t)$ is dependent on the nanoparticle concentration gradient at time $t$ and position $x$ through the Fick's first law, as follows

$$
J_{T}(x, t)=-D \nabla C_{N P}(x, t),
$$

where $D\left[\mathrm{~cm}^{2} / \mathrm{s}\right]$ is the diffusion coefficient, assumed as a constant value for a given fluidic medium, and depending on the size and shape of nanoparticles, as well as the interaction with the solvent and viscosity of the solvent. Unfortunately, the Fick's first law works when applied to steady state systems, namely the concentration will keep constant both along the space and in the time. In our application, the concentration changes during the time, since nanoparticles diffuse along the space by determining different levels of concentrations. For that, we need to consider the Fick's second law. In practice, the diffusion associated with the degradation kinetics yields to the following mass balance:

$$
\frac{\delta C_{N P}(x, t)}{\delta t}=D \frac{\delta^{2} C_{N P}(x, t)}{\delta x^{2}}+b^{\prime} \cdot C_{N P}(x, t),
$$

where $b^{\prime}$ is a kinetic rate constant, and $C_{N P}$ is the drug concentration as transmitted from the previous phase. Due to the thinness of the wall as compared to the overall "compartment" radius, we can use Cartesian coordinates as approximation instead of cylindrical coordinates.

We consider our compartment with a total volume $V$, and a flux going through the two reactors $(i . e ., F)$. Then, we can apply and solve the mass balances that follow directly from (14). The concentration of magnitude $C_{N P}$ corresponds to the nanoparticle dose in the tablet. The mass balance on the first reactor can be modeled as a first order differential equation, that is

$$
\frac{d C_{1}(t)}{d t}=-\left(\frac{2 F}{V}+k\right) C_{1}(t)+\left(\frac{2 F}{V}+k\right) C_{\text {input }}(t),
$$

with the following conditions

$$
C_{1}(t=0)=0, \quad C_{\text {input }}(t)=C_{N P} \cdot \delta(t) .
$$

This leads to the solution for $C_{1}(t)$ as

$$
C_{1}(t)=C_{N P} \cdot \exp [-(k+1 / \tau) t],
$$

where $\tau=\frac{V}{2 F}$. The quantity $\left(C_{N P}-C_{1}(t)\right)$ corresponds to the portion of nanoparticle concentration that will be directly eliminated by the body, without reaching the receiver.

As an analogy to (15), we can write the following mass balance equation:

$\frac{d C_{2}(t)}{d t}=-\left(\frac{2 F}{V}+k\right) C_{2}(t)+\left(\frac{2 F}{V}+k\right) \cdot \exp [-(k+1 / \tau) t]$,

whose solution is

$$
C_{2}(t)=\frac{C_{N P} \cdot t}{\tau} \cdot \exp [-(k+1 / \tau) t],
$$

which represents the concentration of dissolved nanoparticles in the compartment.

As we modelled a scenario as a fluidic medium where nanoparticles are free to move following the diffusion laws until reaching the target cells ${ }^{2}$, they are able to form chemical bindings with them. Thus, from (19), we can obtain the concentration of bindings i.e., $C_{b}(t)$, formed due to the dissolved nanoparticles bound with the receptors, which can be described by the following formula:

$$
C_{b}(t) \cdot u(t-\delta)= \begin{cases}N B \cdot\left[1-\exp \left(-\frac{t-\delta}{\tau}\right)\right], & t>\delta \\ 0, & t \leq \delta\end{cases}
$$

where $N B$ is the maximum number of bindings that can occur i.e., $N B=\max C_{2}(t), \tau[\mathrm{s}]$ is the delay necessary for the binding formation, $\delta[\mathrm{s}]$ is the start time of the reception process that is related to the diffusion delay of the nanoparticles, and $u(t)$ is the Heaviside step function. Figure 3 shows the trend of binding concentration, obtained under the assumption that $N B=100, \delta=5 \mathrm{~s}$, and $\tau=3 \mathrm{~s}$.

\footnotetext{
${ }^{2}$ As an instance, a target cell is a tumor cell that has been previously detected.
} 


\section{IN-BODY MODELING: EXPERIMENTAL EVALUATION AND CHARACTERIZATION}

In this section we will describe the details and features of the nanoparticles, and the solution considered to infer the main features of the interaction between nanoparticles and bodylike fluid solution. Specifically, we will study the morphology of the nanoparticles and any $\mathrm{pH}$ variations, as well as the dielectric constant of the considered solutions.

$\mathrm{Nano}-\mathrm{TiO}_{2}$ (anatase, nanopowder, $99.99 \%$ purity; SigmaAldrich) and an aqueous solution of chlorides of sodium, potassium and calcium in the same concentrations as normal body fluids (Ringer solution, $7.5 \mathrm{ml}$ lactic acid, $5.85 \mathrm{~g}$ $\mathrm{NaCl}, 300 \mathrm{ml} \mathrm{H}_{2} \mathrm{O}$ di grade 2 purity, and $700 \mathrm{ml} \mathrm{H}_{2} \mathrm{O}$ and regulation of the $\mathrm{pH}$ to $6.9-7.0$ ) were used for the experiments. This solution is often used as a physiological solution for in vitro study of biomaterials due to its similarity to the body environment.

Two different $\mathrm{TiO}_{2}$ particle sizes have been selected for the experiments, with the aim to assess how this parameter can guide the interactions between the biological system and specific therapeutic nanostructure. Static immersion test has been performed at $37^{\circ} \mathrm{C}\left( \pm 0.4^{\circ} \mathrm{C}\right)$ by adding the nanoparticles by a rapid single dose. The study has been carried out monitoring the following parameters:

1. the structure and the morphology of the nanoparticles before and after their permanence in the physiological solution for 14 days by SEM (Scanning Electron Microscope) observation and X-ray diffraction analysis;

2. the $\mathrm{pH}$ of the solution containing the nanoparticles at a prefixed time and comparing them to the $\mathrm{pH}$ of the physiological solution maintained in the same condition without any nanoparticles inside. Moreover, experimental determination of the complex dielectric constant of the physiological solutions with different concentrations of nanoparticles (i.e. loadings), comparing the results to the un-loaded physiological solution.

The size of the particles has been measured by Small-angle $\mathrm{X}$-Ray scattering technique, as depicted in Figure 4. According to this study the most frequent radius of the particles is $(i) 40.0 A^{\circ}$ (Sample type 1 ) with a surface-to-volume ratio ${ }^{3}$ equal to $0.1003\left(A^{\circ}\right)^{-1}$, and (ii) $44.0 A^{\circ}$ (Sample type 2 ) with a surface-to-volume ratio equal to $0.1422\left(A^{\circ}\right)^{-1}$. A non-homogeneous particles size has a positive effect during the reactions that occur at the surface of the nanoparticles during the bonding of the drugs and consequently during their distribution and transport.

The particle shape and their surface reactivity can influence the transport properties of other particles in the body environment, and thus affecting the adhesion kinetics of the

\footnotetext{
${ }^{3}$ Also called surface-area-to-volume ratio, and represents the amount of surface area per unit volume of an object or collection of objects. It is frequently used to characterize materials with very small diameter (powder), or very porous or that are otherwise not compact.
}

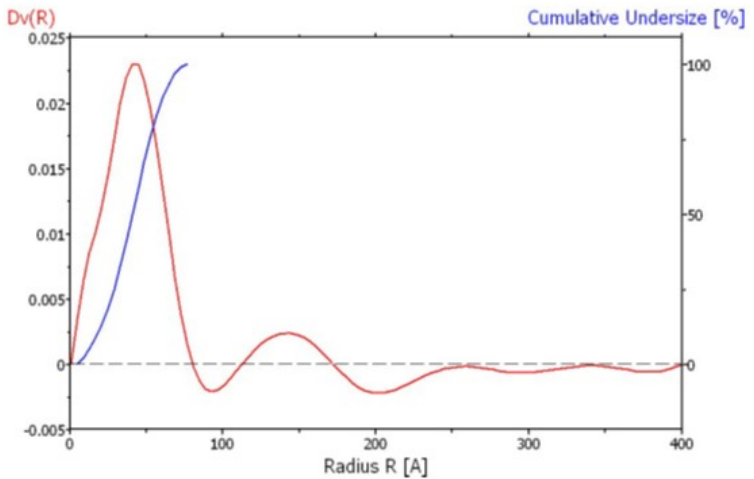

(a)

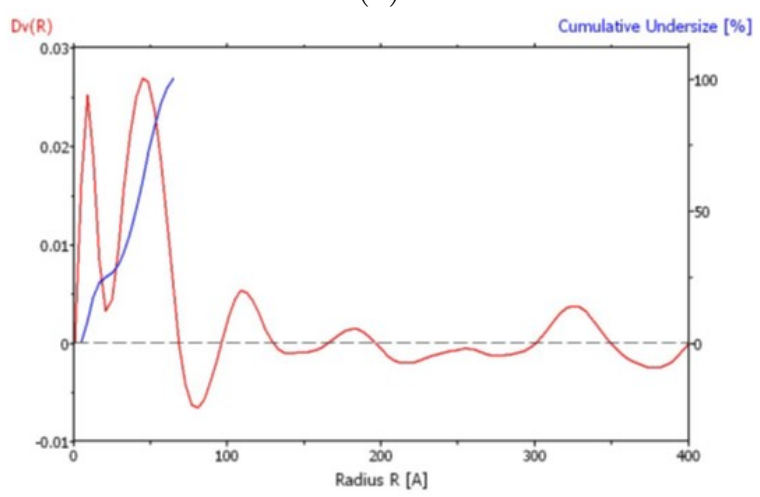

(b)

Figure 4: Size distribution by volume of (a) $40 \mu \mathrm{m}$, and (b) $44 \mu \mathrm{m} \mathrm{TiO}_{2}$ nanoparticles.

therapeutic particles loaded on and their transfer to the favorite position. Figure $5(a)$ and $(c)$ confirm the previously obtained results, that is, the $\mathrm{TiO}_{2}$ nanoparticles used for this study are not perfectly homogeneous as their size concerns, and additionally, because their non-spherical shape can be beneficial to improve drug transport and delivery efficiency as compared to the identical spherical particles.

In such conditions, the difference in curvature between nanoparticles favours an increased loading efficiency and leads obtaining higher attachment to the specific therapeutic particles and a vascular barrier. The nanoparticles show a slightly different microstructure following their permanence in the physiological solution. Indeed, the agglomeration of the nanoparticles takes place (Figure $5(b)$ and $(d)$, and the enhancement of their surface area occurs.

The surface area-to-volume ratio influences the reactions, which occur at the surface; it derives that the particles with a higher surface area have a higher number of reaction sites compared to the particles with a lower surface area, thus resulting in enhanced chemical reactivity. In addition, both $\mathrm{TiO}_{2}$ surfaces show a high tendency to be wetted by the aqueous solution, due to a strong affinity for water.

Apart from the presence of tetragonal Titanium Oxide in both X-ray diffraction spectra, the presence of monoclinic Titanium Hydrogen Oxide has been detected, as shown in 

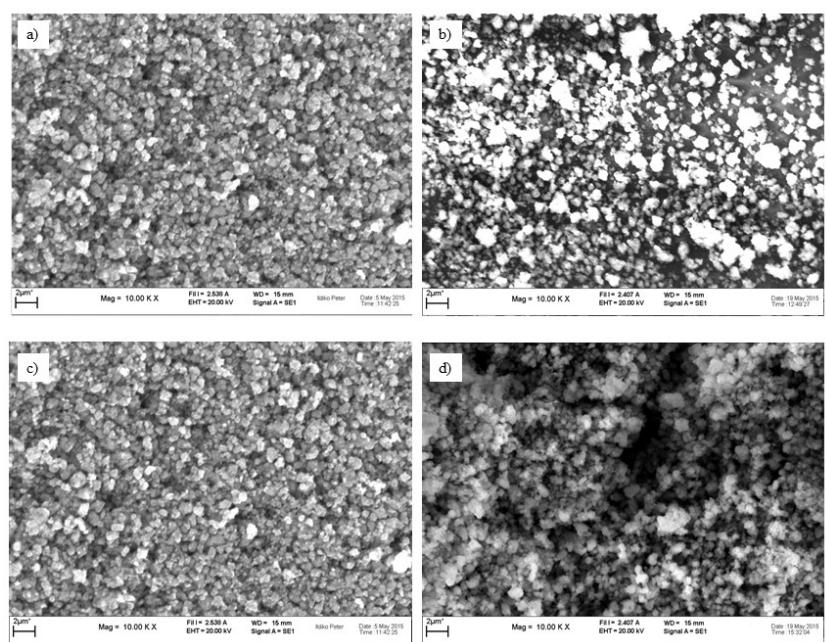

Figure 5: SEM microstructure of the $\mathrm{TiO}_{2}$ nanoparticles at the beginning of the analysis for (a) $40 \mu \mathrm{m}$, and (c) $44 \mu \mathrm{m}$ size, and following the extraction from the physiological solution after 14 days, for (b) $40 \mu \mathrm{m}$, and $(d) 44 \mu m$ size.

Figure 6, following their permanence in the physiological solution. The chemical bond established between the $\mathrm{TiO}_{2}$ nanoparticles and $\mathrm{O}$, and $\mathrm{H}$ atoms coming from the aqueous solution, is an un-stable configuration and confers a hydrophilic character to the nanoparticles, independently form their size, which results to be more reactive towards any chemical compounds.

Finally, by checking the $\mathrm{pH}$ of the solutions for 14 days, no any modification of this parameter was obtained i.e., the $\mathrm{pH}$ value of the simulated body environment remains the same during the experiment $(\mathrm{pH}=6.9-7.0)$. This corresponds to the absence of any release of $T i$ ions in the physiological solution and indicates a non-toxic character of the considered system.

Experimental characterization of the complex dielectric constant of solutions with different $\mathrm{TiO}_{2}$ concentrations [20, 21] has been carried out using 85070D Dielectric Probe Kit (Agilent, now Keysight Technology) attached to a E8361A PNA Network Analyzer $(10 \mathrm{MHz}$ to $67 \mathrm{GHz})$, and compared with the Ringer solution and distilled water reference cases.

A $f_{\text {step }}=10 \mathrm{MHz}$ frequency step between $f_{\min }=60 \mathrm{MHz}$ and $f_{\max }=8 \mathrm{GHz}$ has been considered. Real and imaginary parts are reported in Figure 7 and 8 in the considered frequency band. The values are shown splitting the overall considered frequency band in the interval $[60,200] \mathrm{MHz}$, and from $100 \mathrm{MHz}$ to $8 \mathrm{GHz}$. For the sake of completeness the measured values of the distilled water (also used for the calibration) are also reported.

The dispersion in the considered wide frequency band is basically determined by the Ringer solution's characteristics. A high value of losses in the low frequency range can be observed, that has a minimum around the $f \approx 2 \div 3 \mathrm{GHz}$ range. Above this frequency the imaginary part of $\varepsilon_{\text {eff }}$ asymptot-

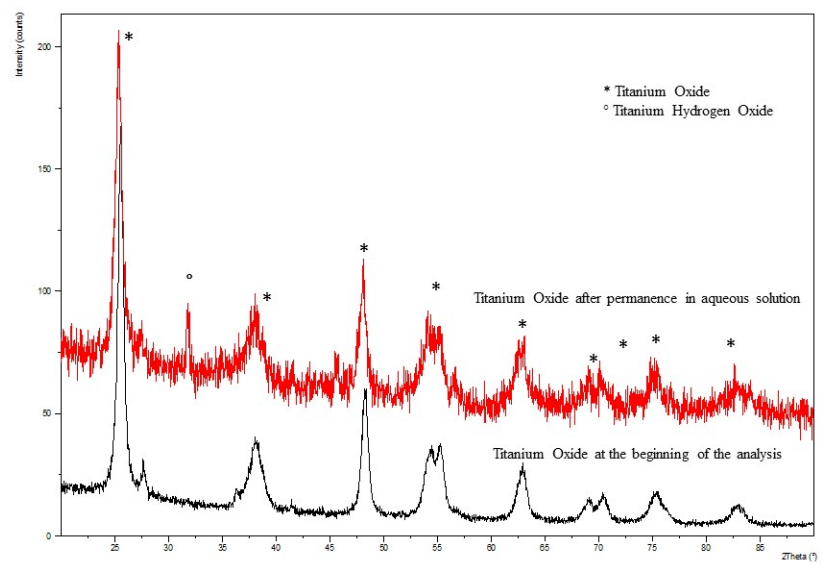

Figure 6: X-Ray diffraction patterns for the starting $\mathrm{TiO}_{2}$ and following the extraction from the physiological solution after 14 days
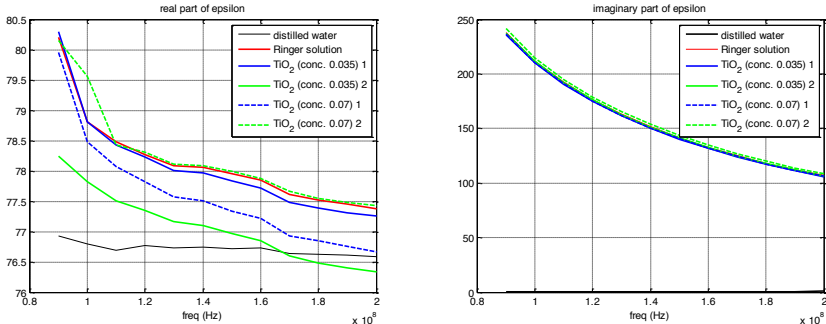

Figure 7: Variation of the real (left) and imaginary (right) part of the complex dielectric constant in the range $[60,200] \mathrm{MHz}$ for different solutions.

ically approaches the reference (i.e., distilled water curve). The real part of the doped liquid has a more significative variation with respect to the imaginary part that is not influenced by the presence of the $\mathrm{TiO}_{2}$ nanoparticles. On the other hand, the real part feels both the concentrations and dimensions of the nanoparticles. This observation allows envisaging changes of the concentration and/or dimensions of the $\mathrm{TiO}_{2}$ nanoparticles based on other considerations depending on the thought-out applications, as for example quantity of the drug to be delivered, characteristics of the targeted organs, etc.

\section{CONCLUSIONS AND FUTURE DIREC- TIONS}

In this paper, we investigated how nanoparticles are injected in the human body for nanomedicine applications. The behavior at the nano-scale is described by means the modeling of the emission, and diffusion processes. Specifically, the one compartment model for oral drug administration is presented and discussed. $\mathrm{TiO}_{2}$ nanoparticles and a biological fluid system as possible carriers to sustain drugs have been studied. 

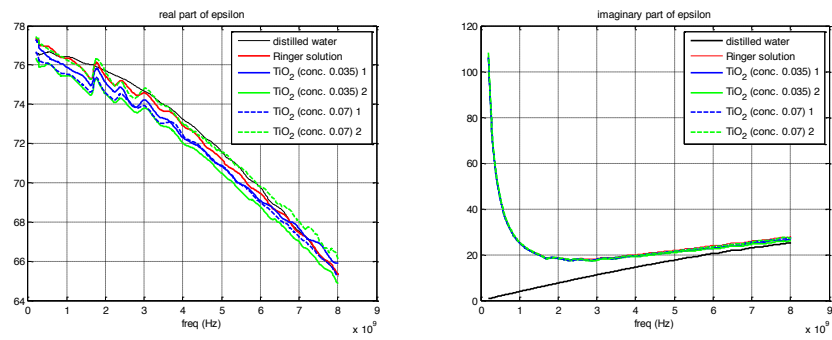

Figure 8: Variation of the real (left) and imaginary (right) part of the complex dielectric constant in the range from $100 \mathrm{MHz}$ to $8 \mathrm{GHz}$ for different solutions.

This research shows the possibility of combining the biocompatibility of $\mathrm{TiO}_{2}$ to the drug delivery features and their use in biomedical field. We observed the complete absence of any release of $T i$ ions in the physiological solution demonstrating, once again, a non-toxic character of the considered system. The particles with a high surface area show a higher tendency to be wetted by the aqueous biological solution and they can support more efficiently any drug loaded on their surfaces.

As future work, we will consider the morphological differences in such a way to match them with specific and different tasks, e.g. based on their properties they can be more suitable for drug delivery or for diagnostic purposes. For the last aspects is of paramount importance to take into account all the specific features and then the specific requirements needed. Furthermore, we are planning to investigate deeply the interactions between the signalling features with the specific system introduced in this work, namely the "invivo-like" environment with nano- $\mathrm{TiO}_{2}$ particles immersed in the Ringer solution.

\section{REFERENCES}

[1] V. Loscrí and A. M. Vegni, "On the affection of the human immune system on a nanoparticulate nanomedicine system," in Proceedings of the 9th International Conference on Body Area Networks BODYNETs, 2014.

[2] S. Svenson and R. K. Prud'homme, Multifunctional Nanoparticles for Drug Delivery Applications: Imaging, Targeting, and Delivery, Springer, Ed., 2012.

[3] R. A. Freitas, "What is nanomedicine?" Nanomedicine: nanotechnology, biology, and medicine, vol. 1, no. 1, pp. 2-9, March 2005.

[4] V. Loscrí and A. M. Vegni, "An acoustic communication technique of nanorobot swarms for nanomedicine applications," NanoBioscience, IEEE Transactions on, vol. PP, no. 99, pp. 1-1, 2015.

[5] V. Loscrí, C. Marchal, N. Mitton, G. Fortino, and A. V. Vasilakos, "Security and privacy in molecular communication and networking: Opportunities and challenges," IEEE Transactions on NanoBioscience, vol. 13, no. 3, pp. 198-207, 2014.

[6] N. Kumar, Handbook of Particulate Drug Delivery, A. S. Publishers, Ed., 2008.

[7] Y. Zhou, Y. Kong, S. Kundu, J. Cirillo, and H. Liang,
"Antibacterial activities of gold and silver nanoparticles against escherichia coli and bacillus Calmette-Guérin," Journal of Nanobiotechnology, vol. 10, 2012.

[8] M. Hossen, K. Kajimoto, H. Akita, M. Hyodo, and H. Harashima, "Vascular-targeted nanotherapy for obesity: Unexpected passive targeting mechanism to obese fat for the enhancement of active drug delivery," J. Control. Release, vol. 163, pp. 101 - 110, 2012.

[9] D. Vllasaliu, C. Alexander, M. Garnett, M. Eaton, and S. Stolnik, "Fc-mediated transport of nanoparticles across airway epithelial cell layers," $J$. Control. Release, vol. 158, pp. 479-486, 2012.

[10] D. Bhumkar, H. Joshi, M. Sastry, and V. Pokharkar, "Chitosan reduced gold nanoparticles as novel carriers for transmucosal delivery of insulin," Pharmaceutical Research, vol. 24, 2007.

[11] J. Cleland, A. Daugherty, and R. Mrsny, "Emerging protein delivery methods," Curr. Opin. Biotechnol., vol. 12, pp. 212-219, 2001.

[12] M. Long and H. Rack, "Titanium alloys in total joint replacement - a materials science perspective," Biomaterials, vol. 19, no. 18, pp. 1621 - 1639, 1998.

[13] M. N. Helmus, D. F. Gibbons, and D. Cebon, "Biocompatibility: Meeting a key functional requirement of next-generation medical devices," Toxicologic Pathology, vol. 36, no. 1, pp. 70-80, 2008.

[14] E. Gultepe, D. Nagesha, S. Sridhar, and M. Amiji, "Nanoporous inorganic membranes or coatings for sustained drug delivery in implantable devices," Advanced Drug Delivery Reviews, vol. 62, no. 3, pp. $305-315,2010$.

[15] I. Peter and M. Rosso, "Study of Ti-enriched CoCrMo alloy for dental application," Access, IEEE, vol. 3, pp. 73-80, 2015.

[16] I. Peter, M. Rosso, A. Toppi, I. Dan, and B. Ghiban, "Investigation on cobalt based alloy modified by titanium for dental application," Archives of Materials Science and Engineering, vol. 61, no. 2, pp. 62-68, June 2013.

[17] W. Utembe, K. Potgieter, A. B. Stefaniak, and M. Gulumian, "Dissolution kinetics of titanium dioxide nanoparticles: the observation of an unusual kinetic size effect," Particle and Fibre Toxicology, vol. 12, no. 11, pp. 1-12, 2015.

[18] J. Schmidt and W. Vogelsberger, "Dissolution kinetics of titanium dioxide nanoparticles: the observation of an unusual kinetic size effect," J Phys Chem B., vol. 110, pp. 3955-3963, 2006.

[19] I. Llatser, E. Alarcon, and M. Pierobon, "Diffusion-based channel characterization in molecular nanonetworks," in Proc. of the 1st IEEE International Workshop on Molecular and Nano Scale Communication (MoNaCom), in conjunction with IEEE INFOCOM, Shanghai (China), April 2011.

[20] J. Bao, C. Davis, and M.Swicord, "Microwave dielectric measurements of erythrocyte suspensions," Biophysical Journal, vol. 66, pp. 2173-2180, 1994.

[21] U. Kaatze, "Complex permittivity of water as a function of frequency and temperature," Journal Chemical Engineering Data, vol. 34, pp. 371-374, 1989. 